\title{
International Journal of Analysis and Applications
}

\section{Differential Equations Models and Their Applications in Metallurgy}

\author{
Ridha Selmi ${ }^{1,3,4, *}$, Muflih Alhazmi ${ }^{1}$, Abir Sboui $^{2,4}$, Hanen Louati $^{1}$, \\ Amel Touati ${ }^{1}$, Hechmi Hattab ${ }^{5,6}$ \\ ${ }^{1}$ Department of Mathematics, College of Sciences, Northern Border University, P.O. Box 1321, Arar, \\ 73222, KSA \\ ${ }^{2}$ Department of Mathematics, Faculty of Sciences and Art (TURAIF), Northern Border University, \\ KSA \\ ${ }^{3}$ Department of Mathematics, Faculty of Sciences of Gabes, 6072, Gabès, Tunisia \\ ${ }^{4}$ Laboratory of partial differential equations and applications (LR03ES04), Faculty of sciences of \\ Tunis, University of Tunis El Manar, 1068 Tunis, Tunisia \\ ${ }^{5}$ FS Sfax, Univercité de Sfax, Route de la Soukra km 4 - Sfax - 3038, Tunisia \\ ${ }^{6}$ ISIM Gabès, Université de Gabès, Cité Erriadh, Zrig, 6072, Gabès, Tunisia \\ *Corresponding author: Ridha.selmi@nbu.edu.sa, ridhaselmiridhaselmi@gmail.com
}

\begin{abstract}
This paper focus on the heat recovery from the metallurgical and mining wastes. We propose and study a new and more realistic mathematical model for heat recovery from molten slag. Our model is based on time delay differential equations. In the theoretical part, we prove that a unique solution exists to the mathematical problem. In the numerical part, we establish an algorithm based on explicit fourth order Runge-Kutta method with delay; the new feature is that the delay must be larger enough than the step of integration. Compared to the classical model (without time delay), the numerical test proves that our model is more efficient and industrially more profitable.
\end{abstract}

\section{Introduction}

In metallurgy, heat recovery from molten slag is nowadays one of the relevant manners to valorize the huge amount of thermal energy provided by such mining waste. This very high temperature liquid

Received: Oct. 7, 2021.

2010 Mathematics Subject Classification. 34K05; 65L03; 65L20; 97 M50.

Key words and phrases. heat transfer; heat recovery from molten slag; delayed convection; mathematical model; time delay-differential equations; Runge-Kutta method; numerical solution. 
slag is refrigerated then solidified by quenching procedure via fluids (gas or liquid), in general. After that, the heat recuperated is used for many purposes that range from industrially to domestically use. Such huge thermal energy can be used for producing electrical energy, or heating buildings, for example. A review paper about this subject is [13]. The report [11] deals with this matter in Australia. The paper [10] presents a global study of the problem, with focus on South Africa mining and metallurgical industry.

In the literature, from mathematical point of view, models dealing with heat recovery from molten slag are formulated using ordinary differential equations that are taking in account only instantaneous properties of the molten slag. Thus, they naturally consider the ordinary time derivative. Such classical models were given by the ordinary differential equation of the form:

$$
\frac{d T}{d t}(t)=f(t, T(t))
$$

where $T$ is the temperature of the molten slag, $t$ is the time variable and $f$ is a given function that describes the process of energy transfer. From physical point of view, as stated in [8], models above are based on the fact that convection and radiation are mainly the two manners of heat dissipation, when liquid slag is quenched. The heat transfer between the quenching fluid and the very hot slag is governed by the energy equality

$$
c V \rho \frac{d T}{d t}(t)=A h\left(T(t)-T_{f}\right)+A \varepsilon \sigma_{0}\left(T^{4}(t)-T_{f}^{4}\right),
$$

where $T$ is the unknown temperature of the molten slag, it depends only on the time $t$. The parameters $V$ and $A$ are respectively the volume and the surface of the molten slag; $T_{f}$ is the fixed temperature of the incoming quenching fluid; $c$ and $\varepsilon$ state for the heat of the alloy and its integrated radiant remittance, $\rho$ is the melt density, $h$ is the heat transfer coefficient of the interface and finally $\sigma_{0}$ refers to the blackbody radiation coefficient.

However, we think that models above are neither realistic nor so much efficient. In fact, from physical and industrial point of view, there are two intersecting issues (a property and a phenomenon) that should be considered:

- Memory effects caused by the viscoelasticity of the molten slag: As for such property, based on physical experiences and chemical analysis the detailed review paper [9] and references therein putted in evidence the viscoelastic response of molten slag, where according to the authors "at high temperatures (which is our case), the time dependent constitutive relations are needed not only for the stress-stress relationship, but also for the heat flux vector" (page 26). Also, they reviewed and discussed "the various existing implicit constitutive models for non-linear viscoelastic materials that can be used to model the rheological characteristics of slag" (page 28). 
- Time delay phenomena occurring in the interconnections of different parts of a system: As for such phenomena, authors in [6] used a space-averaging technique and the method of characteristics to propose a time-delay system modelling the flow temperatures of a heat exchanger; they believe that "time delay phenomena naturally occur in the interconnections of different parts of a system, as propagation of matter is not instantaneous. In particular, it occurs in tubular heat exchangers, which are very common devices in industry". Tubular heat exchangers are exactly the case of quenching systems used for molten slag. In [12], a dual-phase-lagging model of the micro-scale heat conduction is re-derived analytically from the Boltzmann transport equation. Then, based on such model, a delay-advanced partial differential equations governing the micro-scale heat conduction are established, as a more realistic model.

To take in account facts signaled above, we will opt for a model governed by a time-delay equations. Mathematically speaking, this is based on delay differential equations (DDEs), where the derivative of the unknown molten slag temperature $T$ at a time $t$ is expressed by the values of the temperature at previous times that is

$$
\frac{d T}{d t}(t)=f(t, T(t-\tau))
$$

where $T(t-\tau)$ is the delay term that takes in account the memory effects of the system, and $\tau$ is the delay time. In the first chapter of the book [1], it was discussed, in details, how delayed differential equations are a reliable mathematical tool to modelize systems with memory. Based on (1.2), to make the model of heat recovery from molten slag more realistic, we propose the following new governing equation:

$$
c V \rho \frac{d T}{d t}(t)=\left[A h\left(T\left(t-\tau_{1}\right)-T_{f}\right)+A \varepsilon \sigma\left(T^{4}\left(t-\tau_{2}\right)-T_{f}^{4}\right)\right]
$$

where we performed a correction via different time delays $\tau_{1}$ and $\tau_{2}$, respectively in the convective term and the radiation term.

However, to simplify this model, we recall the following: By one hand, as radiation is an electromagnetic wave travelling, heat transfer by radiation occurs at the speed of light. By the other hand, as convection is a phenomenon related to the physical medium, heat transfer by convection takes place at the speed of convective medium. Thus, we are lead to consider that

$$
\tau_{2}<<\tau_{1}
$$

because heat transfer by radiation is faster than heat transfer by convection. Mathematically speaking, we can neglect $\tau_{2}$ compared to $\tau_{1}$. Thus, model (1.3) reads

$$
c V \rho \frac{d T}{d t}(t)=\left[A h\left(T\left(t-\tau_{1}\right)-T_{f}\right)+A \varepsilon \sigma\left(T^{4}(t)-T_{f}^{4}\right)\right]
$$

Above, we mean by "faster" the way to transfer energy from one medium to another in least amount of time. 
From numerical point of view, it is already known, in the literature, that numerical investigation of delayed differential equations is so delicate and some times problematic. Among others, authors in the recent publication [4] discussed several problems that arise while implementing numerical methods to solve delay-differential equations, as overlapping, difficulties with error estimation, discontinuities of solution derivatives and their detection, with focus on the Runge-Kutta methods. Let us explicitly say that, one of serious problems in such investigation is the fact that integration in time variable will be perturbed by some shift effects due to delays. In $[2,7]$, this anomaly was overcome by interpolation. Here, we will take the step of time integration so small compared to the delay. So, the time shift will not be in the interval of integration. This allows us to establish an explicit delayed fourth order Runge-Kutta algorithm (DFOR-K) valid for our proposed model. To the best of our knowledge, our idea is new and original.

The remainder of this paper will be as follows. In section two, we will prove that our model is mathematically well posed; that is a unique solution exists to the corresponding Cauchy problem. Section three deals with the numerical investigation, we will establish an explicit forth order RungeKutta algorithmi, after which numerical test will be presented to validate the model. This paper will be achieved by a closely conclusion.

\section{Functional setting and theoretical results}

In this section, we will prove that a solution to our model exists and it is unique. Following mathematical material in [5], we recall the following.

- The naturel functional setting used to study retarded differential equations is the set of continuous functions mapping the interval $[-r, 0]$ into $\mathbb{R}^{n}, r>0$, denoted by $C=C\left([-r, 0], \mathbb{R}^{n}\right)$, and endowed with the usual norm defined by

$$
|\phi|=\sup _{-r \leq \theta \leq 0}|\phi(\theta)|
$$

for any given function $\phi \in C$.

- If we consider $\sigma \in \mathbb{R}, A \geq 0$ and $x \in C\left([\sigma-r, \sigma+A], \mathbb{R}^{n}\right)$, then for any $t \in[\sigma-r, \sigma+A]$, we let $x_{t} \in C$ be defined by

$$
x_{t}(\theta)=x(t+\theta), \quad-r \leq \theta \leq 0 .
$$

- If $D$ is a subset of $\mathbb{R} \times C, f: D \rightarrow \mathbb{R}^{n}$ is a given function and "." represents the derivative, then we say that the relation

$$
(D D E) \quad \dot{x}(t)=f\left(t, x_{t}\right)
$$

is a delayed (retarded or advanced) differential equation (also said functional differential equation) on $D$ and will denote this equation by $(D D E)$. Equation $(D D E)$ is a very general type 
of equation and includes ordinary differential equations

$$
\dot{x}(t)=f(t, x(t))
$$

whenever $r=0$ (and thus $\theta=0$ ); differential equations in the form

$$
\dot{x}(t)=f(t, x(t), x(t-\tau)), \quad 0 \leq \tau \leq r,
$$

for example which is our case, in this paper.

- A delayed initial value problem is simply a delayed differential equation supplemented by an initial value $\phi$ at $\sigma$ :

$$
(D I V P) \quad\left\{\begin{array}{l}
\dot{x}(t)=f\left(t, x_{t}\right) \\
x_{\sigma}=\phi
\end{array}\right.
$$

where $\sigma \in \mathbb{R}, \phi \in C$ are given.

- A function $x$ is said to be a solution of a delayed differential equation on $[\sigma-r, \sigma+A)$ if there are $\sigma \in \mathbb{R}$ and $A>0$, such that $x \in C\left([\sigma-r, \sigma+A), \mathbb{R}^{n}\right),\left(t, x_{t}\right) \in D$ and $x(t)$ satisfies equation (DDE) for $t \in[\sigma, \sigma+A)$. It follows that $x(\sigma, \phi, f)$ is a solution to the delayed initial value problem $(D I V P)$, that is a solution of $(D D E)$ with initial value $\phi$ at $\sigma$ or simply a solution through $(\sigma, \phi)$, if there is an $A>0$, such that $x(\sigma, \phi, f)$ is a solution of equation $(D D E)$ on $[\sigma-r, \sigma+A)$ and $x_{\sigma}(\sigma, \phi, f)=\phi$.

For interested readers, [5] and [1] are complete references about this topic. Using notation above, we introduce the existence result (Theorem 2.3 page 42 ), in [5]:

Theorem 2.1. Suppose $\Omega$ is an open subset in $\mathbb{R} \times C$ and $f: \Omega \rightarrow \mathbb{R}^{n}$ is continuous, and $f(t, \phi)$ is Lipschitzian in $\phi$ in each compact set in $\Omega$. If $(\sigma, \phi) \in \Omega$, then there is a unique solution of equation $(D D E)$ passing through $(\sigma, \phi)$.

We use theorem above to deal with existence and uniqueness of solution to our model.

(1) Existence of solution: the function $T: t \rightarrow T(t)$ should belong to $C(J)$, so it should be continuous in time $t$ on some time interval $J \subset \mathbb{R}$. This interval will be defined in practice by the duration of the quenching process. Thus, the operator $f: J \times C(J) \rightarrow \mathbb{R}$, such that $f(t, T(t))=a T(t-\tau)+b T^{4}(t)+c$ is continuous in $(t, T)$ as a polynomial in the variable $T$, here we note that there is no explicit dependence of the operator $f$ on the time $t$ and such dependence is implicitly through the temperature $T$. Above, the coefficients $a, b$ and $c$ are given by the parameters of the model after standard computation. So, at this step a solution to our model (1.4) exists.

(2) Uniqueness of solution: Also, as a polynomial, the operator $f$ is Lipschitzian in $T$ in each compact set in $J \times C(J)$. Thus, if we supplement our model by the initial value $T_{\sigma}=T_{i n}$, theorem above asserts that our model has a unique solution $T\left(\sigma, T_{i n}, f\right)$ passing through $\left(\sigma, \phi=T_{i n}\right)$. Here, we note that $T_{i n}$ is the temperature of the molten slag before the 
beginning of the quenching process and we recall that $T_{\sigma}$ is defined by $T_{\sigma}(\theta)=T(\sigma+\theta)$, $-r \leq \theta \leq 0, r>0$, so that $T_{\sigma} \in C$ as it is a constant function of time equal to $T_{\text {in }}$ everywhere. The value of $\sigma$ can be taken equal to zero, without loss of generality.

\section{Numerical investigation}

3.1. The delayed fourth order Runge-Kutta algorithm. The classical fourth Runge-Kutta method consists in integrating a differential equation of the form

$$
\frac{d T}{d t}(t)=f(t, T(t))
$$

using the formula: $t_{n+1}=t_{n}+h$, where $h$ is the time step and

$$
T_{n+1}=T_{n}+\frac{1}{6}\left(K_{1}+2 K_{2}+2 K_{3}+K_{4}\right), \quad \text { for } n=1,2, \ldots, m-1,
$$

where

$$
\begin{aligned}
& K_{1}=h f\left(t_{n}, T_{n}\right) \\
& K_{2}=h f\left(t_{n}+\frac{h}{2}, T_{n}+\frac{K_{1}}{2}\right) \\
& K_{3}=h f\left(t_{n}+\frac{h}{2}, T_{n}+\frac{K_{2}}{2}\right) \\
& K_{4}=h f\left(t_{n}+h, T_{n}+K_{3}\right)
\end{aligned}
$$

and $m$ is the number of integration's steps.

Let us consider the following delay differential equation:

$$
\begin{aligned}
\frac{d T}{d t}(t)=f(t, T(t-\tau), T(t)) & =\frac{1}{c V \rho}\left[A h\left(T(t-\tau)-T_{f}\right)+A \varepsilon \sigma\left(T^{4}(t)-T_{f}^{4}\right)\right] \\
& =\alpha T(t-\tau)+\beta T^{4}(t)+\gamma
\end{aligned}
$$

where $\alpha=(6 * h) /(\rho * c * d), \beta=(6 * \varepsilon * \sigma) /(\rho * c * d)$ and $\gamma=\left(\alpha * T_{f}\right)+\left(\beta *\left(T_{f}^{4}\right)\right)$, and we approximate the molten slag grains by spheres of radius $d / 2$. We claim that the diameter $d$ and thus the surface $A$ can be adjustable, by industrial methods to increase or decrease the transfer interface between molten slag and the fluid. In reference ( [8]), the coefficients $\alpha, \beta$ and $\gamma$ are calculated based the physical parameters of model (1.1), to obtain

$$
\begin{aligned}
& \alpha=-5.20810^{-6} \\
& \beta=-4.62610^{-3} \\
& \gamma=38381.568 .
\end{aligned}
$$

For sake of comparison of our delayed model to the non delayed model introduced in ( [8]), we are using the same coefficients in our numerical study. Also, as in ( [8]), we take the initial temperature of the slag to be $T_{\text {in }}=T(0)=1773 \mathrm{~K}$. Thus, in our following numerical investigation, we will consider 
the delayed initial value problem

$$
\left\{\begin{array}{l}
\frac{d T}{d t}(t)=-5.20810^{-6} T(t-\tau)-4.62610^{-3} T^{4}(t)+38381.568 \\
T_{\text {in }}=1773 \mathrm{~K} .
\end{array}\right.
$$

If the delay $\tau$ is larger enough than the step of integration, the above fourth Runge-Kutta method can be extended to a fourth Runge Kutta algorithm with delay by expressive constants $\tilde{K}_{1}, \tilde{K}_{2}, \tilde{K}_{3}$ and $\tilde{K}_{4}$ with the delayed form of the $f(t, T(t-\tau), T(t))$. That is

$$
\begin{aligned}
\tilde{K}_{1} & =h f\left(T\left(t_{n}\right)\right)=h\left[\alpha T\left(t_{n}-\tau\right)+\beta T^{4}\left(t_{n}\right)+\gamma\right] ; \\
\tilde{K}_{2} & =h f\left(T\left(t_{n}\right)+\frac{1}{2} \tilde{K}_{1}\right) \\
& =h\left[\alpha\left(T\left(t_{n}\right)+\frac{1}{2} \tilde{K}_{1}\right)_{\left(t_{n}-\tau\right)}+\beta\left(T\left(t_{n}\right)+\frac{1}{2} \tilde{K}_{1}\right)^{4}+\gamma\right] \\
& =h\left[\alpha\left(\left(T\left(t_{n}-\tau\right)+\frac{1}{2}\left(\tilde{K}_{1}\right)_{\left(t_{n}-\tau\right)}\right)+\beta\left(T\left(t_{n}\right)+\frac{1}{2} \tilde{K}_{1}\right)^{4}+\gamma\right],\right.
\end{aligned}
$$

where

$$
\begin{aligned}
& \left(\tilde{K}_{1}\right)_{\left(t_{n}-\tau\right)}=h f\left(T\left(t_{n}-\tau\right)\right) \\
= & h\left[\alpha T\left(t_{n}-2 \tau\right)+\beta T^{4}\left(t_{n}-\tau\right)+\gamma\right] ; \\
\tilde{K}_{3}= & h f\left(T\left(t_{n}\right)+\frac{1}{2} \tilde{K}_{2}\right) \\
= & h\left[\alpha\left(T\left(t_{n}\right)+\frac{1}{2} \tilde{K}_{2}\right)_{\left(t_{n}-\tau\right)}+\beta\left(T\left(t_{n}\right)+\frac{1}{2} \tilde{K}_{2}\right)^{4}+\gamma\right] \\
= & h\left[\alpha\left(\left(T\left(t_{n}-\tau\right)+\frac{1}{2}\left(\tilde{K}_{2}\right)_{\left(t_{n}-\tau\right)}\right)+\beta\left(T\left(t_{n}\right)+\frac{1}{2} \tilde{K}_{2}\right)^{4}+\gamma\right],\right.
\end{aligned}
$$

where

$$
\begin{aligned}
\left(\tilde{K}_{2}\right)_{\left(t_{n}-\tau\right)} & =h f\left(T\left(t_{n}-\tau\right)+\frac{1}{2}\left(\tilde{K}_{1}\right)_{\left(t_{n}-\tau\right)}\right) \\
& =h\left[\alpha\left(T\left(t_{n}-2 \tau\right)+\frac{1}{2}\left(\tilde{K}_{1}\right)_{\left(t_{n}-2 \tau\right)}\right)+\beta\left(T\left(t_{n}-\tau\right)+\frac{1}{2}\left(\tilde{K}_{1}\right)_{\left(t_{n}-\tau\right)}\right)^{4}+\gamma\right] \\
\left(\tilde{K}_{1}\right)_{\left(t_{n}-2 \tau\right)} & =h f\left(T\left(t_{n}-2 \tau\right)\right)=h\left[\alpha T\left(t_{n}-3 \tau\right)+\beta T^{4}\left(t_{n}-2 \tau\right)+\gamma\right]
\end{aligned}
$$

and

$$
\begin{aligned}
\tilde{K}_{4} & =h f\left(T\left(t_{n}\right)+\tilde{K}_{3}\right) \\
& =h\left[\alpha\left(T\left(t_{n}\right)+\tilde{K}_{3}\right)_{\left(t_{n}-\tau\right)}+\beta\left(T\left(t_{n}\right)+\tilde{K}_{3}\right)^{4}+\gamma\right] \\
& =h\left[\alpha\left(\left(T\left(t_{n}-\tau\right)+\left(\tilde{K}_{3}\right)_{\left(t_{n}-\tau\right)}\right]+\beta\left(T\left(t_{n}\right)+\tilde{K}_{3}\right)^{4}+\gamma\right],\right.
\end{aligned}
$$


where

$$
\begin{aligned}
\left(\tilde{K}_{3}\right)_{\left(t_{n}-\tau\right)} & =h f\left(T\left(t_{n}-\tau\right)+\frac{1}{2}\left(\tilde{K}_{2}\right)_{\left(t_{n}-\tau\right)}\right) \\
& =h\left[\alpha\left(T\left(t_{n}-2 \tau\right)+\frac{1}{2}\left(\tilde{K}_{2}\right)_{\left(t_{n}-2 \tau\right)}\right)+\beta\left(T\left(t_{n}-\tau\right)+\frac{1}{2}\left(\tilde{K}_{2}\right)_{\left(t_{n}-\tau\right)}\right)^{4}+\gamma\right] \\
\left(\tilde{K}_{2}\right)_{\left(t_{n}-2 \tau\right)} & =h f\left(T\left(t_{n}-2 \tau\right)+\frac{1}{2}\left(\tilde{K}_{1}\right)_{\left(t_{n}-2 \tau\right)}\right) \\
& =h\left[\alpha\left(T\left(t_{n}-3 \tau\right)+\frac{1}{2}\left(\tilde{K}_{1}\right)_{\left(t_{n}-3 \tau\right)}\right)+\beta\left(T\left(t_{n}-2 \tau\right)+\frac{1}{2}\left(\tilde{K}_{1}\right)_{\left(t_{n}-2 \tau\right)}\right)^{4}+\gamma\right] \\
\left(\tilde{K}_{1}\right)_{\left(t_{n}-3 \tau\right)} & =h f\left(T\left(t_{n}-3 \tau\right)\right)=h\left[\alpha T\left(t_{n}-4 \tau\right)+\beta T^{4}\left(t_{n}-3 \tau\right)+\gamma\right] .
\end{aligned}
$$

Then,

$$
T_{n+1}=T_{n}+\frac{1}{6}\left(\tilde{K}_{1}+2 \tilde{K}_{2}+2 \tilde{K}_{3}+\tilde{K}_{4}\right), \quad \text { for } n=1,2, \ldots, m-1,
$$

where $t_{n+1}=t_{n}+h, h$ is the time step and $m$ is the number of integration's steps.

We will name the algorithm (3.4)-(3.10) the delayed fourth order Runge-Kutta algorithm that we will denote by (DFOR-K). From the above computation, it is clear that since equation (3.3) is with one constant delay $\tau$, the (DFOR-K) depends on the delay of time equals to $4 \tau$ via $\left(\tilde{K}_{1}\right)_{\left(t_{n}-3 \tau\right)}$, in the last line of (3.9). This proves the following theorem.

Theorem 3.1. Let $\tau$ be the delay in equation (3.3) and $h$ be the integration step in the (DFOR-K) (3.4)-(3.10). If $\tau \geq h$, then the (DFOR-K) (3.4)-(3.10) is a valid algorithm. Moreover, it depends on delays $\tau, 2 \tau, 3 \tau$ and $4 \tau$.

3.2. Numerical results. We present two numerical results. The first concerns the comparison between the model with delay, that it is physically more realistic, and the one without delay. The second result consists in establishing the variation of the heat transfer as a function of the exchange surface between the molten slag and the quenching fluid.

In Figure 1 and Table 1, we fix the thermal exchange surface of the molten slag with the fluid used for the recovery of the heat to be $A=200$ units of the sphere of diameter $d$ modeling the grains of the molten slag and we present the decrease in the temperature of the molten slag as a function of time, with an integration step Timestep $=0.5 \mathrm{~s}$, a delay $\tau=4 *$ Timestep, where the number of steps of integration is $m=40$. So, the simulation is in a time equal to $20 \mathrm{~s}$. Clearly, we can see that, when taking into account the delay, the decrease in temperature is more important, which implies a saving of the time while transferring heat from the molten slag. Also, we see that the behaviour of the temperature as a function of time presents some asymptote that seems to be the same for the two models (without and with delay). This leads to think about the optimal time to spend in the heat recovery process, in industry. 
Table 1. Table for the delay $\tau=4$ Pastemps $=4$ steps of integration. pastemps $=0.5 \mathrm{~s}, \mathrm{~A}=200$ sphere unities, number of integration's steps $m=40$.

\begin{tabular}{|c|c|c|c|}
\hline Iteration & Times (s) & Delayed temperature & Not delayed temperature \\
\hline 0 & 0.0000000000000000 & 00000000000 & 00000000 \\
\hline 1 & 0.50000000000000000 & 1496.3627665694594 & 1487.6130483245572 \\
\hline 2 & 1.0000000000000000 & 1228.2200943503881 & 26885185 \\
\hline 3 & 1.5000000000000000 & 1011.7376907722477 & 1078.339 \\
\hline 4 & 2.0000000000000000 & 843.90934123125589 & 931.22909171791252 \\
\hline 5 & 2.5000000000000000 & 715.08690804465118 & 812.09212122248778 \\
\hline 6 & 3.0000000000000000 & 616.41586854571949 & 715.41277990356048 \\
\hline 7 & 3.5000000000000000 & 540.85202877542747 & 636.85701598717617 \\
\hline 8 & 4.0000000000000000 & 482.97008411070431 & 572.97465271184217 \\
\hline 9 & 4.5000000000000000 & 438.62089061602052 & 520.99665252349666 \\
\hline 10 & 5.0000000000000000 & 404.63356920917545 & 478.68924422998879 \\
\hline 11 & 5.5000000000000000 & 378.58332275097030 & 444.24459090912433 \\
\hline 12 & 6.0000000000000000 & 358.61453884968427 & 416.19650804817417 \\
\hline 13 & 6.5000000000000000 & 343.30637504591556 & 393.35426511243531 \\
\hline 14 & 7.00 & 331 & 519 \\
\hline 15 & 7.5000000000000000 & 322.57283358988946 & 359.59628591699783 \\
\hline 16 & 8.0000000000000000 & 315.67440783 & 075992033 \\
\hline 17 & 8.5000000000000000 & 310.38531550573572 & 337.19755665628946 \\
\hline 18 & 9.0000000000000000 & 306.33005421868648 & 329.00648616513820 \\
\hline 19 & 9.5000000000000000 & 303.22076278047979 & 322.33372710777883 \\
\hline 20 & 10.000000000000000 & 300.83675480171695 & 316.89774647706361 \\
\hline 21 & 10.500000000000000 & 299.00883657838762 & 312.46924901901673 \\
\hline 22 & 11.000000000000000 & 297.60728872134200 & 308.86147204479352 \\
\hline 23 & 11.500000000000000 & 296.53265440274487 & 305.92228806723062 \\
\hline 24 & 12.000000000000000 & 295.70867813729103 & 303.52777670666762 \\
\hline 25 & 12.500000000000000 & 295.07689267128490 & 301.57699142269786 \\
\hline 26 & 13.000000000000000 & 294.59246909462632 & 299.98769831306481 \\
\hline 27 & 13.500000000000000 & 294.22103526397939 & 298.69290600508037 \\
\hline 28 & 14.000000000000000 & 293.93623652689627 & 297.63803951277356 \\
\hline 29 & 14.500000000000000 & 293.71786551843422 & 296.77863839112746 \\
\hline 30 & 15.000000000000000 & 293.55042824457450 & 296.07848181653878 \\
\hline
\end{tabular}




\begin{tabular}{|l|l|l|l|}
\hline 31 & 15.500000000000000 & 293.42204466040243 & 295.50806134363791 \\
\hline 32 & 16.000000000000000 & 293.32360570627554 & 295.04333682354223 \\
\hline 33 & 16.500000000000000 & 293.24812697431037 & 294.66472295529155 \\
\hline 34 & 17.000000000000000 & 293.19025313632625 & 294.35626369636543 \\
\hline 35 & 17.500000000000000 & 293.14587796555929 & 294.10495969758477 \\
\hline 36 & 18.000000000000000 & 293.11185298858754 & 293.90022039122590 \\
\hline 37 & 18.500000000000000 & 293.08576409385415 & 293.73341762396927 \\
\hline 38 & 19.000000000000000 & 293.06576024566658 & 293.59752201192765 \\
\hline 39 & 19.500000000000000 & 293.05042215001640 & 293.48680668521655 \\
\hline 40 & 20.000000000000000 & 293.03866155349442 & 293.39660593217508 \\
\hline
\end{tabular}

Our second numerical test focus on the effect of the variation of the contact surface between the quenching fluid and the molten slag surface on the heat transfer rate from this molten slag. It is clear that increasing this exchange surface has as an effect to increase the speed of the heat recovery from the molten slag, in both cases of modeling with delay (Figure 2) and without delay (Figure 3). Also, it is clear that while passing from surface $A=100$ to surface $A=200$, temperature decreases faster than while passing from $A=200$ to $A=300$ an so on, for the same abscise of time, see for example for $t=5 \mathrm{~s}$ of (Figure 2). This leads to think about the optimally profitable surface that should be considered in industrial protocols. Moreover, the behavior of the temperature as a function of time presents some horizontal asymptote that should define the stopping time of the heat recovery procedure, in practice.

Combining the above two statements, as a result, even more heat exchange speed is gained one takes into account the delay and increases the surface of the contact.

Remark 3.1. The fact when passing from surface $A=100$ to surface $A=200$, temperature decreases faster than when passing from $A=200$ to $A=300$ an so on, may be du to the hypothesis we made in the beginning that is $\tau_{2}<<\tau_{1}$. In a forthcoming paper, we will deal with the model (1.3) and discuss the situation based on this model that we think to be a compete one. 


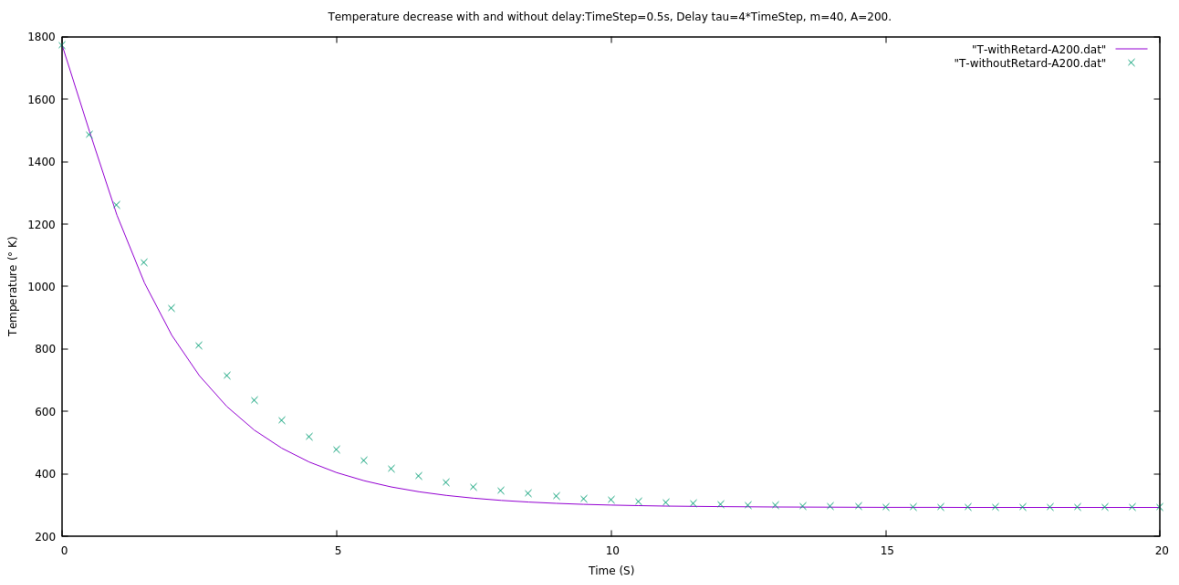

Figure 1. Decrease in the temperature, $\tau=4 *$ timestep, timestep $=0.5 s$ and $m=40$.

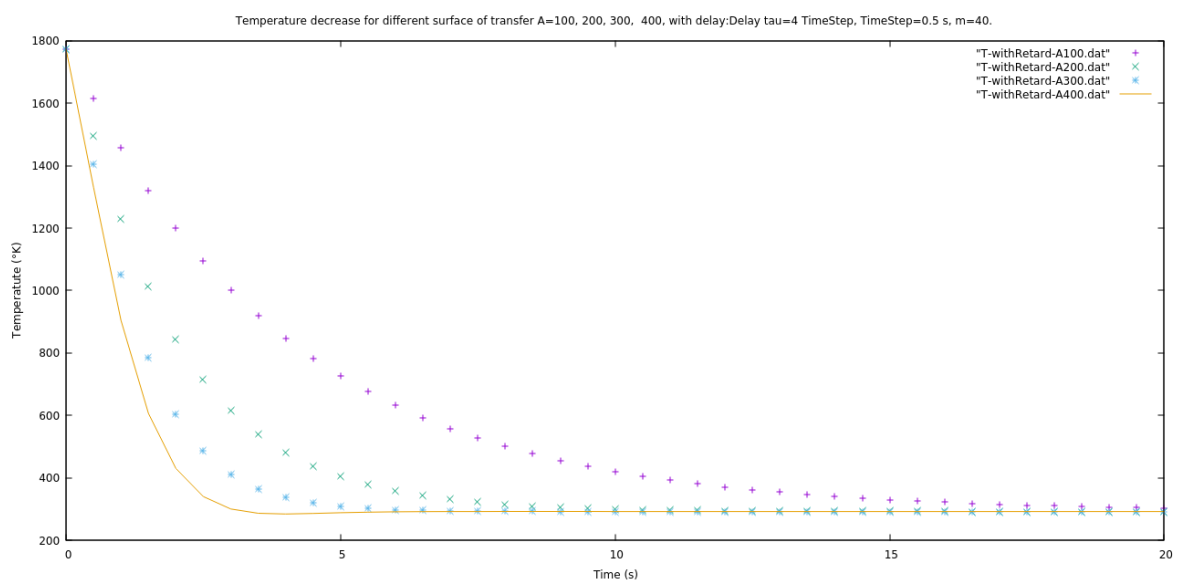

Figure 2. Decrease in the temperature for different surface of heat transfer: $A=100$, 200, 300, \& 400; $\tau=4 *$ timestep, timestep $=0.5$ s and $m=40$.

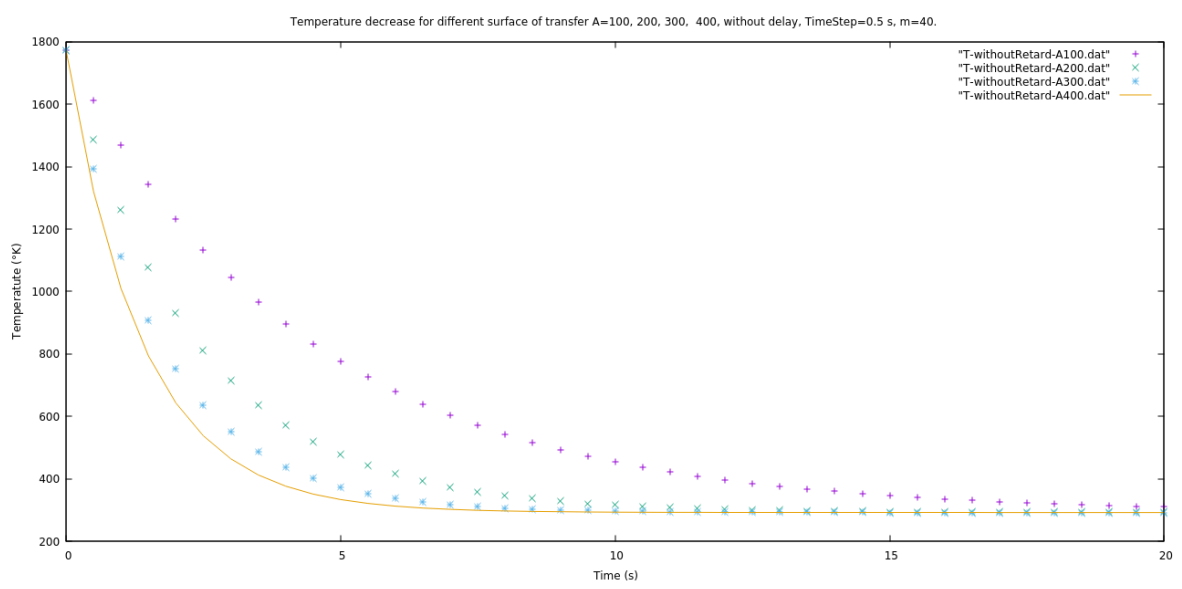

Figure 3. Decrease in the temperature for different surface of heat transfer: $A=100$, $200,300, \& 400$, without delay, timestep $=0.5 s, m=40$. 


\section{Conclusion}

Heat recovery and re-use of the huge amount of thermal energy offered by the very high temperature of the metallurgical and mining waste, in molten slag allow to built a circular economic model and prevent environmental damages. Such industrial procedure should be an efficient process both in the sense of duration of heat recovery and the optimal logistic protocol. In this paper, we proposed a time delayed model that is more realistic then the instantaneous one. Our model is mathematically well posed and numerically solvable using a variant of Runge-Kutta method which is popular. First, we proved that our model is more efficient compared to the classical one, in the sense that the heat recovery is faster; this saves time in industry. Also, we proved that there should exist both a stopping time to the quenching process and an optimal surface of the contact between the molten slag and the quenching fluid, so that the heat recovery procedure is maximally profitable, in industry.

Acknowledgement: The authors extend their appreciation to the Deputyship for Research \& Innovation, Ministry of Education in Saudi Arabia for funding this research work through the project number "3400_2020_IF".

Conflicts of Interest: The author(s) declare that there are no conflicts of interest regarding the publication of this paper.

\section{References}

[1] O. Arino, M.L. Hbid, E. Ait Dads, Delay differential equations and applications, Springer, Netherlands, (2006).

[2] N. A. Ayunni Sabri, M. bin Mamat, Solving delay differential equations (DDEs) using Nakashima's 2 stages $4^{\text {th }}$ order Pseudo-Runge-Kutta Method, World Appl. Sci. J. 21 (2013), 181-186.

[3] L. Cheng, M. Xu, L. Wang, From Boltzmann transport equation to single-phase-lagging heat conduction, Int. J. Heat Mass Transfer. 51 (2008), 6018-6023. https://doi.org/10.1016/j.ijheatmasstransfer.2008.04.004.

[4] A. S. Eremin, A. R. Humphries, A. A. Lobaskin, Some issues with the numerical treatment of delay differential equations, AIP Conf. Proc. 2293 (2020), 100003. https://doi.org/10.1063/5.0027149.

[5] J. K. Hale, S. M. Verduyn Lunel, Introduction to functional differential equations, Springer Verlag, Berlin, 1993.

[6] S. Hamze, E. Witrant, D. Bresch-Pietri, C. Fauvel, Estimating heat-transport and time-delays in a heat exchanger, in: 2018 IEEE Conference on Control Technology and Applications (CCTA), IEEE, Copenhagen, 2018: pp. 1514-1519. https://doi.org/10.1109/CCTA.2018.8511359.

[7] F. Ismail, R. A. Al-Khasawneh, A. S. Lwin, M. B. Suleiman, Numerical treatment of delay differential equations by Runge-Kutta method using Hermite interpolation, MATEMATIKA: Malaysian J. Ind. Appl. Math. 18 (2002), 79-90

[8] C. Liu, H. Wu, J. Chang, Research on a class of ordinary differential equations and application in metallurgy, in: R. Zhu, Y. Zhang, B. Liu, C. Liu (Eds.), Information Computing and Applications, Springer Berlin Heidelberg, Berlin, Heidelberg, 2010: pp. 391-397. https://doi.org/10.1007/978-3-642-16339-5_52.

[9] M. Massoudi, P. Wang, A brief review of viscosity models for slag in coal gasification, DOE/NETL-2012/1533, National Energy Technology Laboratory, Pittsburgh, PA, 2011.

[10] E. Matinde, G.S. Simate, S. Ndlovu, Mining and metallurgical wastes: a review of recycling and re-use practices, J. South. Afr. Inst. Min. Metall. 118 (2018), 825-844. https://doi.org/10.17159/2411-9717/2018/v118n8a5. 
[11] D. Xie, Y. Pan, R. Flann, B. Washington, S. Sanetsis, J. Donnelley et al., Heat recovery from slag through dry granulation, in: 1st CSRP Annual Conference. Melbourne (Australia), vol. CSIRO Minerals, pp. 29-30, 2007.

[12] M. Xu, L. Wang, Dual-phase-lagging heat conduction based on Boltzmann transport equation, Int. J. Heat Mass Transfer 48 (2005), 5616-5624. https://doi.org/10.1016/j.ijheatmasstransfer.2005.05.040.

[13] H. Zhang, H. Wang, X. Zhu, Y.-J. Qiu, K. Li, R. Chen and Q. Liao, A review of waste heat recovery technologies towards molten slag in steel industry, Appl. Energy 112 (2013), 956-966. https://doi .org/10.1016/j . apenergy . 2013.02 .019$. 